\title{
CONTRIBUIÇÃO PARA AS INFORMAÇÕES EPIDEMIOLÓGICAS DE MALÁRIA NO ESTADO DE SÃO PAULO, BRASIL
}

\author{
Dalva Marli Valério Wanderley, José Carlos Rehder de Andrade e Maria \\ José Chinelatto Pinheiro Alves
}

\begin{abstract}
Os autores apresentam os primeiros resultados obtidos através de um sistema de processamento eletrônico das informações constantes na Ficha de Investigação Epidemiológica de Malária no Estado de São Paulo. Os casos de malária foram detectados através da busca passiva, observando-se que na sintomatologia predominaram queixas referentes a febre, cefaléia e dores musculares. A população de enfermos em São Paulo é constituída, em $74 \%$ dos casos, por pessoas residentes no Estado que se deslocaram para a Regiāo Amazônica tendo em vista desenvolver atividades relacionadas principalmente com a extração de minerais, transporte de carga e agropecuária.

Os autores discutem as relaçóes entre a notificação de casos e o seu encaminhamento pelos serviços de saúde, ressaltando a necessidade de avaliar os métodos de divulgação utilizados que visam sensibilizar a população e a categoria médica ante o progressivo aumento da incidência da malária no Estado de São Paulo.
\end{abstract}

Palavras chaves: Malária. Vigilância epidemiológica. Controle da malária. Epidemiologia.

A vigilância epidemiológica da malária no Estado de São Paulo tem por objetivo final evitar a introdução da enfermidade e a reinstalação da transmissão da mesma, através da deteç̧ão precoce de portadores da doença procedentes principalmente da Amazônia Legal, onde a endemia encontra-se em expansão nos últimos anos 56 .

Para tanto, o conhecimento da população sujeita ao risco de contrair malária torna-se fundamental para o êxito das ações de controle. Estudos anteriores ${ }^{3}, 46$ têm demonstrado ser a mesma constituida por indivíduos do sexo masculino, na faixa etária economicamente produtiva que se deslocam para áreas endêmicas do País.

Tendo como propósito aperfeiçoar o sistema de vigilância epidemiológica da endemia no Estado, foi proposto o estudo de informações - importantes e até então não analisadas - constantes da Ficha de Investigação Epidemiológica, atualmente em uso pela Superintendência de Controle de Endemias (SUCEN).

Nesse sentido foi desenvolvido um sistema de processamento eletrônico visando o armazenamento das informações em computador. O presente trabalho apresenta os resultados preliminares obtidos através deste sistema, a partir da análise das notificações de casos de malária registrados em 1985.

Endereço para correspondência: Dra. Dalva Marli Valério Wanderley - Superintendência de Controle de Endemias (SUCEN) da Secretaria de Estado da Saúde. Rua Paula Souza 166 - 01027 São Paulo, SP, Brasil.

Recebido para publicação em $06 / 10 / 86$.

\section{MATERIAL E MÉTODOS}

O sistema de processamento eletrônico foi desenvolvido em 1985, através de contrato firmado junto à Companhia de Processamento de Dados do Estado de São Paulo (PRODESP), utilizando-se o Sistema de Investigação Científica.

Partindo-se da premissa que qualquer informação pode ser expressa quer por uma variável numérica, quer por um descritor, foi desenvolvido um sistema hierárquico em 4 níveis parametrizados, ou seja, dinâmico, onde toda informação pode ser incluída, alterada ou excluída do modelo inicial, através da simples atualização de um descritor, em qualquer um dos 4 niveis.

No primeiro nivel definiu-se o universo de estudo, ou seja o armazenamento de todas as informaçōes do "Programa de Erradicação da Malária". Definiu-se, no segundo nivel, todos os subconjuntos formadores deste universo. No momento, o único subconjunto existente no sistema é a Ficha de Investigação Epidemiológica. Em etapas posteriores, poderão ser absorvidos outros grupos de informação. No terceiro nivel foram definidas as questões que comporiam cada subconjunto. Neste modelo foram desenvolvidas 38 questões que abrangem as informações constantes da Ficha de Investigação Epidemiológica (dados pessoais, laboratoriais e epidemiológicos). Em um quarto nivel definiu-se as diferentes possiveis respostas de cada questão expressas quer sob a forma de variáveis contínuas, quer sob a forma de descritores.

O sistema permite que qualquer informação ou conjunto de informações sejam cruzados com qualquer 
Wanderley DMV, Andrade JCR, Alves MJCP. Contribuição para as informações epidemiológicas de malária no Estado de São Paulo, Brasil. Revista da Sociedade Brasileira de Medicina Tropical 20: 143-146, Jul-Set, 1987.

outra informação ou conjunto de informações, através de um terminal de vídeo. O sistema informará dentro de um universo pesquisado quais são os elementos que atenderão as condições solicitadas.

Desta forma, foi desenvolvido um modelo de protocolo para onde as informações coletadas nas 2.201 notificações de casos de malária de 1985 foram transcritas, procedendo-se então, a atualização do banco de dados.

Até o momento, encontram-se disponiveis para análise um total de 2.092 protocolos, que compõem o universo de dados utilizados no presente estudo.

\section{RESULTADOS}

A deteç̧ão de casos de malária no Estado de São Paulo é constituída principalmente pela busca passiva $(98,4 \%)$ onde a SUCEN é responsável pela notificação de $65,4 \%$ dos'casos (Fig. 1), ao lado de

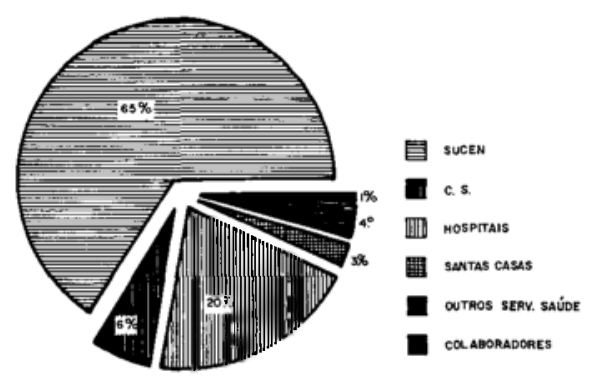

FIguRa I: Procadâncio do notificaģăo dos cosos de malária. Eslado de Sōo Poulo, 1985

Hospitais (20,4\%) e Centros de Saúde $(6,4 \%)$. A maior concentração de doentes foi observada na Grande São Paulo, responsável pelo atendimento de $42,7 \%$ dos casos detectados.

O Plasmodium vivax está presente em 1.284 casos $(61,4 \%)$, o Plasmodium falciparum em 595 $(28,5 \%)$ e ambas as espécies em $213(10,1 \%)$ casos. A sintomatologia é carácterizada principalmente por febre, cefaléias e dores musculares. Quadros assintomáticos foram relatados em $33(1,6 \%)$ pacientes.
Antecedente malárico foi constatado em 1.256 pacientes, demonstrando que a freqüência da malária por $P$. vivax e $P$. falciparum são bastante próximas, 37,0 e $39,6 \%$, respectivamente. Por outro lado, a malária anterior é referida em $85,5 \%$ dos casos nos últimos seis meses que antecederam a malária atual (Tabela 1). No tocante a medicação utilizada na cura destes episódios maláricos anteriores, constatou-se que $21,2 \%$ dos pacientes não souberam informar. Entre as respostas positivas, as drogas mais freqüentes são a cloroquina, primaquina e o quinino. Chama a atenção o fato de $89,7 \%$ dos pacientes afïrmarem não ter tomado medicação profilática.

A população de doentes de malária é constituída em 74,0\% por individuos residentes em São Paulo, sendo os demais $(26,0 \%)$, residentes em outros Estados, ou seja, estavam em trânsito quando da confirmação do diagnóstico.

No tocante a ocupação, garimpeiros, motoristas de transporte de carga e trabalhadores agricolas estiveram presentes em $17,7 \%, 15,4 \%$ e $12,1 \%$ das fichas, respectivamente. Com referência aos deslocamentos dos pacientes verificou-se que: $1.646(79,1 \%)$ haviam viajado para a Amazônia Legal, $323(15,5 \%)$ para outras regiões do País (incluindo-se aqueles que têm residência fixa em Estados da Amazônia Legal e que para cá vieram), $11(0,5 \%)$ para o exterior, 13 $(0,6 \%)$ para outros municipios do Estado de São Paulo que não o de residência e $99(4,3 \%)$ não relataram viagens. Dos pacientes que viajaram $90,7 \%$ indicaram contatos com portadores de malária no local para onde se deslocaram e/ou residem, incluindo os que viajaram para o exterior.

O estudo do motivo de viagem em 1.993 doentes demonstrou que: $25,7 \%$ estavam relacionados com a extração de minerais, $13,3 \%$ com a atividade de transporte de carga e $10,9 \%$ com projetos agropecuários. Importante ressaltar ainda entre estes doentes, os $12,1 \%$ que referiram o lazer como motivo da viagem (Fig. 2). Entretanto, há que se considerar que este grupo inclui parcela dos pacientes com residência fixa em Estados da Amazônia Legal e que vieram para São Paulo, em visita a parentes.

Tabela 1 - Pacientes com antecedente malárico, segundo a espécie apresentada e o tempo decorrido em dias.

\begin{tabular}{lcccrrr}
\hline Espécie de & \multicolumn{2}{c}{ Tempo em dias } & \multicolumn{3}{c}{ Total } \\
Plasmódio & $<30$ & $31-180$ & $181-365$ & $>366$ & \multicolumn{1}{c}{ No } & $\%$ \\
\hline P. vivax & 105 & 284 & 49 & 27 & 265 & 37,0 \\
P. falciparum & 193 & 244 & 32 & 28 & 497 & 39,6 \\
Associado & 23 & 48 & 8 & 8 & 87 & 6,9 \\
Não Sabe & 45 & 103 & 21 & 38 & 207 & 16,5 \\
\hline Total & 366 & 679 & 110 & 101 & 1256 & 100,0 \\
$\quad(\%)$ & $(29,1)$ & $(54,1)$ & $(8,8)$ & $(8,0)$ & & \\
\hline
\end{tabular}

Fontè: SUCEN. 


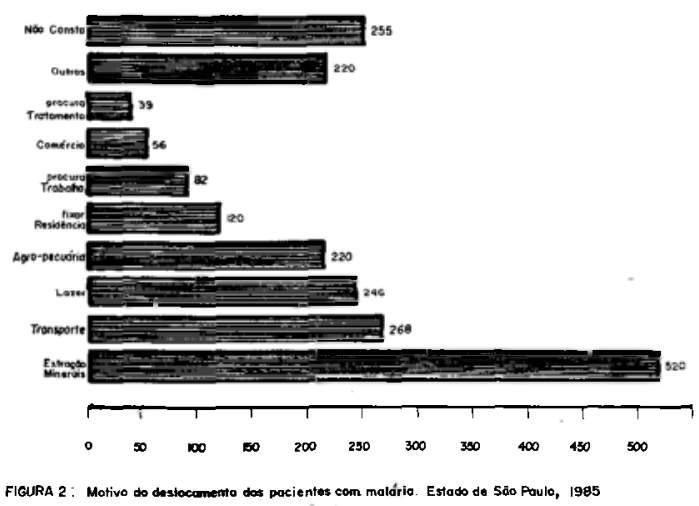

O motivo do retorno dos indivíduos com malária para o Estado de São Paulo em $56,4 \%$ dos casos é a condição de residência. A informação, meio de transporte utilizado, disponivel em $58,0 \%$ dos casos, indica que $46,0 \%$ destes se utilizaram de transporte rodoviário, com predominio de ônibus e caminhão.

Vale considerar que $58,9 \%$ dos pacientes procuraram algum atendimento médico no Estado, antes da confirmação do diagnóstico da malária em laboratório da SUCEN, representado por Centros de Saúde, Hospitais, médico particular, Santas Casas ou outros serviços. Tal informação torna-se pertinente quando se analisa a forma como os pacientes tomaram conhecimento da SUCEN, onde 50,4\% referem ter sido através de serviços de saúde. Por outro lado, ainda com relação ao conhecimento que os pacientes têm da SUCEN, apenas $0,6 \%$ (12 casos) afirmaram que souberam através de jornal, cartaz e/ou rádio/TV (Fig. 3).

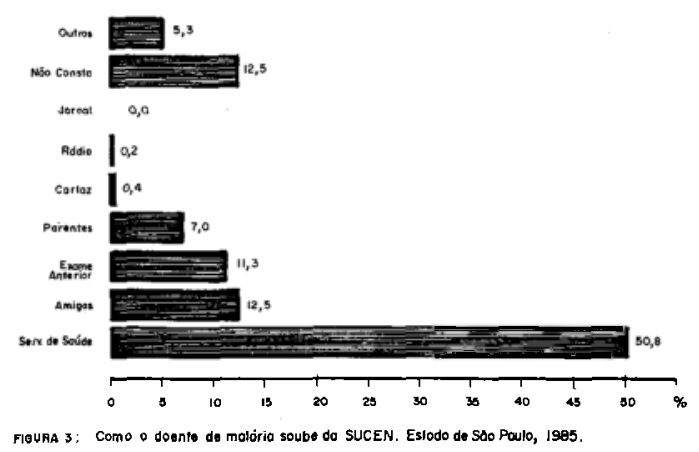

Quanto ao destino do paciente de malária, $76,7 \%(1.605)$ receberam atendimento ambulatorial e foram dispensados com a orientação de retorno para avaliação da cura e $23,3 \%$ (487) foram encaminhados para hospitalização ou encontravam-se internados no momento do diagnóstico.

\section{DISCUSSÃO}

Tendo em vista a tendência crescente da incidência da malária no pais, e o conseqüente reflexo desta situação para o Estado de São Paulo, estudos visando o conhecimento da população de risco tornam-se prioritários, com o objetivo de propiciar melhor direcionamento da vigilância epidemiológica.

A análise da origem da notificação dos casos quando comparada com a passagem prévia do doente por algum serviço de saúde $e$ a forma de como relata ter tomado conhecimento dos serviços da SUCEN, aponta para uma supernotificação atribuída a esta última. Esta informação foi importante para que se procedesse uma discussão sobre o significado da notificação de casos entre os técnicos de um órgão vertical de controle. Assim, o simples encaminhamento do paciente por qualquer serviço de saúde deve ser considerado como notificação deste serviço, independente de atendimento médico. Quando o paciente dirige-se passivamente à rede de laboratório da SUCEN, a partir de conhecimento prévio do Serviço, ou tiver sido detectado em busca ativa de casos, o notificante é a própria SUCEN.

$\mathrm{O}$ antecedente malárico referido em $60,0 \%$ dos pacientes (Tabela 1) levanta dois pontos importantes. Em primeiro lugar, a mobilidade dos portadores de malária atendidos em São Paulo assume característica de circulação, ou seja, deslocamento freqüente para fora do lugar de residência com eventual retorno ${ }^{2}$. Em segundo, a freqüência com que os pacientes referem antecedentes de malária por $P$. falciparum $(39,6 \%)$, maior do que os $37,0 \%$ que referem $P$. vivax.

Este achado embora diferente dos percentuais de participação com que estes dois tipos de malária incidem no Estado de São Paulo6, aproximaram-se daqueles verificados no Brasil $^{1}$, respectivamente $48,3 \%$ e $50,4 \%$.

A ocupação referida pelos indivíduos nem sempre corresponde ao motivo que os levaram a se deslocar em direção às áreas de transmissão de malária. Assim, 371 indivíduos referiram ocupação de garimpeiro, embora 520 tenham relatado como motivo da viagem a atividade de extração de minerais. Tais observações são válidas também para outras categorias profissionais.

O percentual de 56,4 indivíduos com malária que indicaram como motivo da vinda para o Estado de São Paulo a condição de residência, apresenta-se subestimado uma vez que esta informação não constou em $20,7 \%$ dos protocolos, inclusive quando comparado com a condição de trânsito, onde $74,3 \%$ dos pacientes indicaram São Paulo como o Estado de residência.

Outro ponto importante a considerar é a necessidade de avaliação dos métodos de divulgação que vêm sendo utilizados na sensibilização da população e da categoria médica. Enquanto o primeiro grupo não tem sido atingido pelos meios de comunicação de massa, o segundo grupo deveria ser objeto de um trabalho de conscientização de maior impacto, na medida em que pelo menos $58,9 \%$ dos doentes de 
Wanderley DMV, Andrade JCR, Alves MJCP. Contribuição para as informações epidemiológicas de malária no Estado de São Paulo, Brasil. Revista da Sociedade Brasileira de Medicina Tropical 20: 143-146, Jul-Set, 1987.

malária recebem algum tipo de atendimento médico, antes que seja confirmado o diagnóstico hemoscópico de malária pela SUCEN.

As informações divulgadas e analisadas no presente estudo, geradas através do sistema de processamento, ora em uso, não se esgotam neste trabalho, indicando uma série de evidências que necessitam ser investigadas.

\section{SUMMARY}

This paper is concerned with the initial results obtained through an electronic processing system using the data of the Malaria Epidemiological Investigation Card of the State of São Paulo, 1985. Detection of malaria patients was made by passive search the most frequent symptoms being fever, headhache and muscular pain. Seventy four per cent of malaria cases in São Paulo had worked at sometime in a mine, truckage or farming in the Amazonian Region. Relationships between notification and the patient orientation by the health services are discussed. Proper evaluation and adequate divulgation methods are needed in order to interest in the problem not only in the medical stimulate category but also among the people as the incidence of malaria is increasing in the State of São Paulo.
Key Words: Malaria. Epidemiological surveillance. Malaria control. Malaria epidemiology.

\section{REFERÊNCIAS BIBLIOGRÁFICAS}

1. Ministério da Saúde. Superintendência de Campanhas de Saúde Pública. Relatório anual. Brasília, 1983.

2. Prothero RM. Doença e mobilidade: uma questão negligenciada em epidemiologia. In: Seminário sobre Transmissāo e Controle de Doenças Tropicais no Processo de Migração Humana, Brasília, 1982. Anais. Brasilia, p. 169-81, 1982.

3. Secretaria de Estado da Saúde. Superintendência de Controle de Endemias - SUCEN. Relatório sobre avaliaçāo epidemiológica do Programa de Erradicação da Malária no Estado de São Paulo. 26 p. (Documento mimeografado), 1984.

4. Secretaria de Estado da Saúde. Superintendência de Controle de Endemias - SUCEN. Relatório sobre avaliação epidemiológica do Programa de Erradicação da Malária no Estado de São Paulo. 21 p. (Documento mimeografado), 1985.

5. Tauil PL. Malária: agrava-se o quadro da doença no Brasil. Ciência Hoje, 11 : 58-64, 1984.

6. Wanderley DMV, Andrade JCR, Meneguetti LC, Chinelatto MJ, Dutra AP. Malária no Estado de São Paulo, Brasil, 1980 a 1983. Revista de Saúde Pública (São Paulo) 19: 28-36, 1985. 